\title{
Interactive Specification of Flexible User Interface Displays
}

\author{
SCOTT E. HUDSON and SHAMIM P. MOHAMED \\ University of Arizona
}

\begin{abstract}
One of the problems with conventional UIMSs is that very often there is no graphical way to specify interfaces. This paper describes OPUS, the user interface editor of the Penguims UIMS. This system allows the presentation component of graphical user interfaces to be specified interactively in a graphical notation without explicit programming. The Penguims UIMS supports an underlying model of computation based loosely on spreadsheets. In particular, it supports incremental computations based on a system of equations (one-way constraints) over a set of named values (spreadsheet cells). These equations are used to provide immediate feedback at all levels of the interface. They are used to incrementally determine the position and dynamic appearance of the individual interactor objects that make up the interface. They are also used to connect the presentation directly to underlying application data thereby supporting semantic feedback. The OPUS user interface editor employs a special graphical notation for specifying the presentation component of a user interface. This notation allows the power of the underlying computational model to be expressed simply and quickly. The resulting presentations are very flexible in nature. They can automatically respond to changes in the size and position of display objects and can directly support derivation of their appearance from application data objects.
\end{abstract}

Categories and Subject Descriptors: D.2.2 [Software Engineering]: Tools and Techniques-user interfaces; D.2.6 [Software Engineering]: Programming Environments-interactive; D.2.m [Software Engineering]: Miscellaneous-rapid prototyping; 1.3.6 [Computer Graphics]: Methodology and Techniques

General Terms: Human Factors, Languages

Additional Key Words and Phrases: Constraint systems, direct manipulation, end-user programming, interface builders, user interface management systems

\section{INTRODUCTION}

An important goal of the Penguims UIMS project is to support the specification of sophisticated direct manipulation interfaces $[11,15,27,28]$ by nonprogrammers. The OPUS user interface editor is the first step in this direction. OPUS (the On-line Penguims User-interface Specifier) allows the presentation component of a Penguims-based user interface to be specified in a powerful graphical notation with little or no programming. This notation provides a carefully

This work was supported in part by the National Science Foundation under grants IRI-8702784 and CDA-8822652.

Authors' address: Department of Computer Science, University of Arizona, Tucson, AZ 85721.

Permission to copy without fee all or part of this material is granted provided that the copies are not made or distributed for direct commercial advantage, the ACM copyright notice and the title of the publication and its date appear, and notice is given that copying is by permission of the Association for Computing Machinery. To copy otherwise, or to republish, requires a fee and/or specific permission.

(C) 1990 ACM 1046-8188/90/0700-0269 $\$ 01.50$ 
designed set of primitive building blocks that can be used to express most common screen layout constructs. The notation also allows interactor objects taken from an object-oriented toolkit to be positioned and controlled based on information derived from underlying application objects. Finally, the notation allows complex relationships between inputs and outputs to be specified easily.

In the next section the architecture of the Penguims UIMS is briefly introduced and its relationship to OPUS will be considered. Section 3 introduces and motivates the graphical notation that is the central focus of this paper. Section 4 considers related work. Section 5 describes experience gained using the system, and Section 6 finally presents conclusions.

\section{SYSTEM ARCHITECTURE}

In order to clarify the environment in which the OPUS editor operates, this section provides a brief introduction to the architecture and implementation of the Penguims UIMS and explains how OPUS interfaces to Penguims.

\subsection{ARTKit}

Both Penguims and OPUS are implemented in $\mathrm{C}++$ and make use of an extensible object-oriented user interface toolkit-the Arizona Retargetable Toolkit (ARTKit). ARTKit is written in $\mathrm{C}$ and $\mathrm{C}++$ and is designed to run on top of several different host window systems including the $\mathrm{X}$ window system [25] and SunView [30]. ARTKit provides a variety of interactor objects (sometimes called widgets [21]), including buttons, sliders, text entry fields, menus, and so forth. ARTKit also supplies sophisticated redraw control and event dispatch facilities that make the creation of diverse new interactor object classes easier. For example, experimental interactors making use of gesture input [10] and semantic snapping [14] have recently been added to the toolkit.

\subsection{Penguims}

The Penguims UIMS provides an interpreter, which creates and manages ARTKit interactor objects and the semantic relationships between them. Like the NoPumpG system [19,34], Penguims supports a computational model based loosely on spreadsheets. 'The primary construct of the system is the cell. Penguims cells, such as conventional spreadsheet cells, contain a value and an optional equation. If present, the equation specifies how the cell's value is derived from the value of other cells. A set of these equations form a system of one-way constraints. The Penguims system continuously solves these constraints in an efficient incremental fashion to determine dynamically the visibility, position, and other drawing attributes of each interactor object.

Although one-way constraints are not as powerful as more general multiway constraints $[1,4,18]$ they are much easier to control and form a very powerful underlying mechanism for managing screen layout (one-way constraints are also employed in $[23,29]$ ). Because cell values are constantly updated on the basis of constraints, the resulting interfaces generally provide good dynamic feedback that reflects the state of the interface and application. This feedback is an important factor in the support of direct manipulation interfaces. In addition, since complex equations (expressed in a Pascal-like expression language) can be ACM Transactions on Information Systems, Vol. 8, No. 3, July 1990. 
used, it is possible to produce feedback that reflects not only the geometric properties of the interface, but also the semantic properties of the underlying application domain.

Unlike conventional spreadsheet cells, which are placed in a rectangular array, Penguims cells are grouped into objects. A Penguims object is simply a group of named cells. Penguims objects may also contain an interface section that relates the object to one or more interaction objects appearing on the screen or to one or more application data objects.

Although eventually all Penguims code will be specified interactively, the system also supports a conventional textual specification language to aid in code interchange. As an example, consider the following segment of Penguims code:

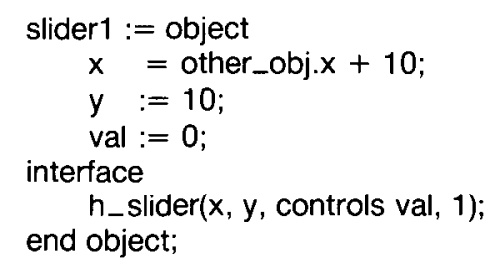

This interface description defines an object: slider1, which contains three cells, $x, y$, and val. The declaration of a cell consists of a name followed either by the assignment of an initial value (using $:=$ ) or by an equation (using $=$ ). In this case $x$ has been given an equation; $y$ and val have only been assigned initial values. In general, within an object cells are referred to by name. Reference to nonlocal cells is also allowed using a dot notation (i.e., other_obj.x refers to the cell $\mathbf{x}$ within the object named other_obj).

In addition to these three cells, slider1 is related to (or owns) one interface object, a horizontal slider. Interface objects are declared in the interface section by giving an interactor or application object class name followed by a set of parameters. These parameters determine how the interface object is related to the cells of the owning Penguims object and whether various parts of the interface object provide inputs, outputs, or both. Each parameter is associated with a different aspect of the interactor object. In this case, the first two parameters are associated with the object's screen position, the third parameter is associated with the value of the slider (the position of the slider's thumb), and the final parameter controls the visibility of the object on the screen.

Each parameter may be coded as a cell name, as the keyword controls followed by a cell name, or as an expression that evaluates to a constant. If a cell name is coded, this indicates that the dynamically changing value of the cell should control this aspect of the interface object. In other words, this part of the interface object produces output based on the (changing) value of the cell but does not accept input. The keyword controls indicates that this part of the interface object is to accept input from the user and that this input is to control the value of the given cell. Finally, when a constant expression is coded, it provides an initial value for the parameter, but no association with a cell is established. In general, particular interactor classes may place restrictions on the form of its parameters (cell, constant, or controls cell) based on whether it can support input, output, both, or neither. 
In the example above, the value of the first two parameters (associated with the position of the slider on the screen) are determined by the cells $x$ and $y$ respectively. However, the third parameter (associated with the slider's value) is used to control the value of the cell val. Finally, the fourth parameter (associated with the visibility of the slider) is assigned an initial value of 1 .

Note that if the third parameter of the slider had not been coded as controls, the slider would have become an output-only interactor, moving only in response to changes in the val cell but not responding to user inputs. However, with the given specification, the slider instead accepts user input and this input determines the value of the val cell.

Some interaction objects need to execute code in response to events. For example, button objects perform specific actions when they are pressed. To support this, the Penguims system provides a special form of data that encapsulates imperative code-code values. Code values may need to be invoked on demand by an interaction object or an application routine in order to modify the state of one or more Penguims objects.

As an example, the object defined below declares one code value and associates it with the cell action. This value is then passed as a parameter to a button interface object that invokes the code whenever the simulated button is pressed by the user. In this case the action modifies the icn_state cell, which in turn causes a new bitmap image to be used for the button.

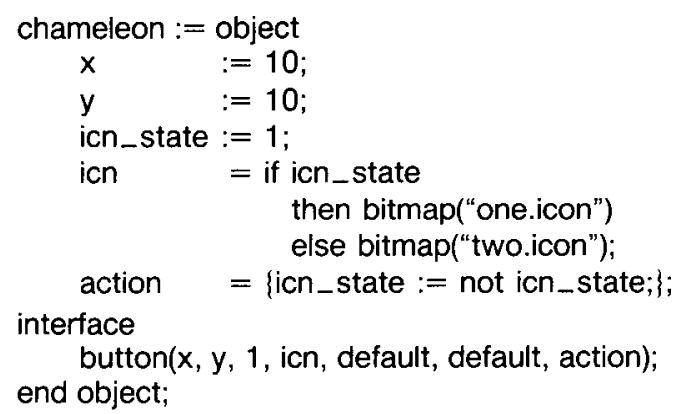

Code values are enclosed in braces, \{ \}. The names appearing within these braces are bound in the context of the object in which they appear (even though they may be executed in a different context). Consequently, Penguims code values are very similar to Smalltalk "block" values [8].

The update of values controlled by equations in the Penguims system is performed in a very efficient manner. The system uses an update algorithm that is both incremental and lazy. After a given update, it only evaluates equations that might change the value of a cell, and it never evaluates an equation until the value is actually needed directly or indirectly to create a display or communicate with the application (see below). The algorithm used is a simplified version of the update algorithm described in [13], which is in turn based on the update algorithm originally developed for use in the Higgens UIMS [12].

Application code written in $\mathrm{C}$ or $\mathrm{C}++$ can be linked with a Penguims interface in several ways. Application programs can provide new functions and procedures that can be invoked directly by Penguims evaluation rules or code. These 
functions and procedures are installed by listing them in a table and linking them with the Penguims object code. In addition, more sophisticated two-way communications between the application and interface are supported by allowing new interface object types to be created. These object types can be used in the interface section of a Penguims object to perform input and output to and from the application in a manner analogous to the input and output performed by interactor objects. Notification of changed values between the application and interface is managed using an active value mechanism similar to the ones found in $[22,26]$. New application interface object types are added to the system by making table entries for creation, destruction, and notification routines and then linking these routines with the Penguims object code.

\subsection{OPUS}

While the textual language accepted by the Penguims UIMS is very powerful and relatively simple, it is still more cumbersome than we might like. In particular, it still has many of the drawbacks of conventional languages-the textual notation is abstract in nature and relies on a highly structured textual syntax that is not visually related to resulting interface. In addition, although the language processor is very fast, it still requires separate edit and translate/execute steps that are not interactive in nature. The OPUS interface editor is designed to eliminate the need for even simple textual specifications like those noted above, replacing them instead with a graphical notation constructed in an interactive environment.

OPUS operates as a separate program that generates Penguims code. However, as described in Section 3.3, it enables the user immediately to try out a partially specified user interface at any time by invoking the Penguims system as a separate process. This is done directly from a menu in the OPUS editor and requires only a fraction of a second. As a result it appears to the user that Penguims is built into OPUS even though the two are separate programs.

Figure 1 shows an example interface being created with the OPIJS editor. The system operates very much like a conventional drawing program. A palette on the left allows the selection of interactor objects and other components of the graphical notation, which may be positioned and manipulated in the large drawing window to the right. The palette also provides control buttons to quit the OPUS session, to try out interactively an interface, to create Penguims code, and to save and restore OPUS specification files.

\section{A GRAPHICAL NOTATION}

In this section we consider the graphical notation used in the OPUS editor. This notation is designed to express the objects and constraint equations needed in the presentation of a user interface. In particular, it specifies a set of interactor objects and the constraints that position them on the screen.

\subsection{Basic Constructs}

The size and position of each interactor object is determined by a bounding rectangle (even if the interactor has a more complicated shape). Each edge of the bounding rectangle determines one coordinate value (top, bottom, left, or right) 


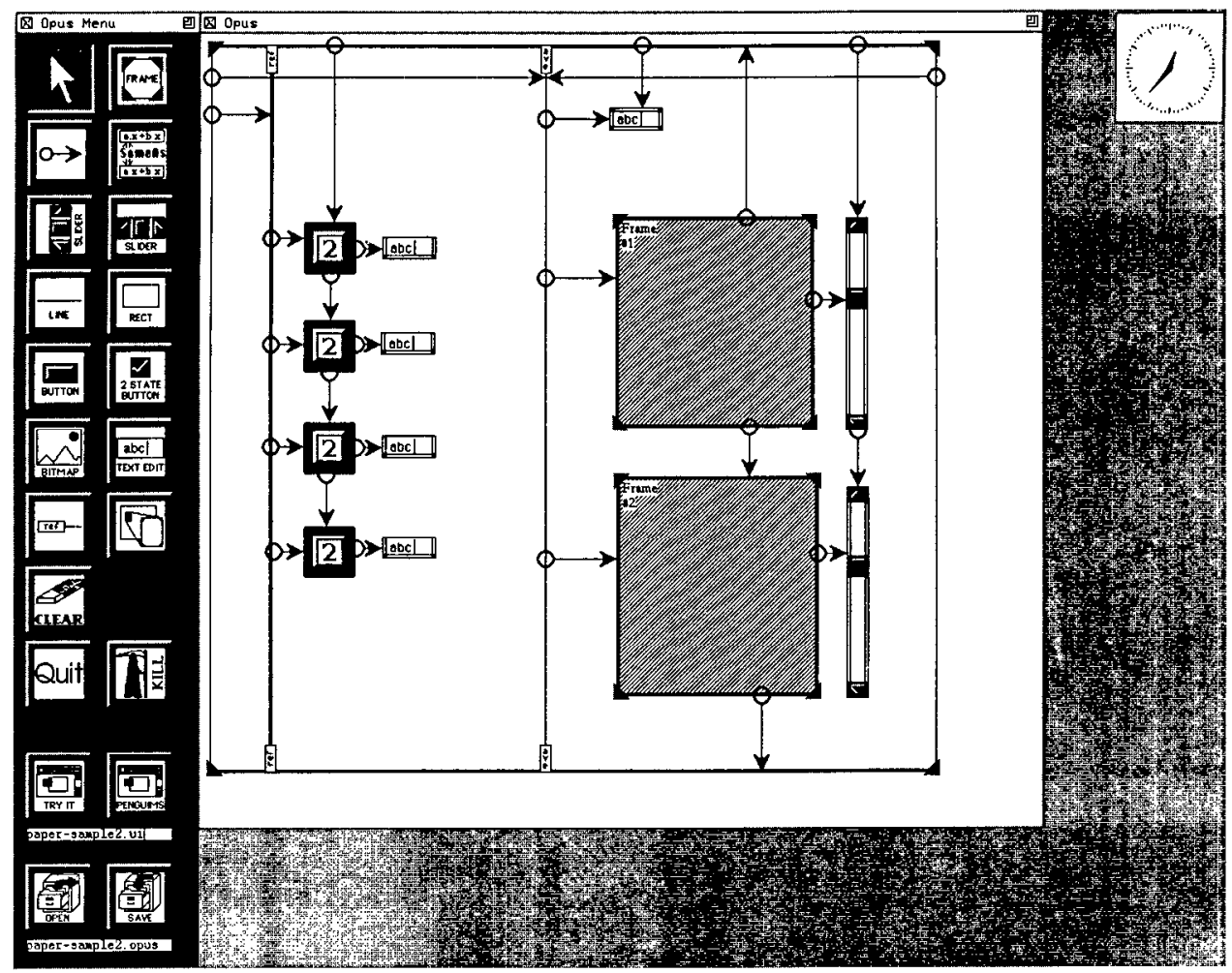

Fig. 1. An interface being created in OPUS.

for the object. The primary task of the graphical notation is to specify a constraint or initial value for each edge of each interactor's bounding rectangle, hence to specify the size and position of each object.

Each edge of a rectangle used in the graphical notation may either be free (having only been assigned an initial value) or may be constrained by an equation. In general, free edges are drawn as a single line; constrained edges are drawn as double lines. This allows quick visual identification of which components can have new constraints attached to them and which are already constrained. Certain interactor classes have internal constraints. For example, the width and height of bitmap or icon objects is determined by the bitmap image used to construct them. In these cases, the bottom and right edges are automatically doubled to indicate the presence of this internal constraint.

The OPUS editor uses four basic notational constructs: Frames, Reference Lines, Constrainls, and Inleractor Objects. The appearance and action of each of these constructs is described below.

- Frames are rectangles that provide a frame of reference for some grouping within the interface. Objects are logically placed within frames; typically, their placement is expressed as constraints derived from the sides of the frame. The frame does not appear in the generated interface but serves as a point of reference to help define relationships between other elements of the interface. 
Frames come in two varieties: simple open frames and hierarchical composition frames. As shown in Figure 2 an open frame is drawn as a rectangle with shaded triangles at each corner. An open frame surrounds each interface specification and is used to compose the interface into larger groupings in a hierarchical fashion. Composition frames are used as place holders for other groupings that form components of the larger layout. To break the interface into manageable sections, the grouping associated with a composition frame is normally displayed in a separate editing window and, as shown in Figure 3, composition frames are drawn filled with a grey pattern.

By using frames as a hierarchical composition agent it is possible to incorporate interface fragments taken from a library of common predefined components into a larger interface. Constraining the objects of each logically related group to conform to the size and position of a frame makes it easy to incorporate the group into a larger interface in a size and position independent manner.

- Reference lines are horizontal or vertical lines attached to frames. Each reference line specifies a single coordinate value that can be used to determine the positions of other objects. They are modeled after the reference lines sometimes used in drafting or technical drawing. In this context, a draftsman will sometimes draw a very thin line that can be used to create a precise alignment of other elements of the drawing. Once the alignment is performed, this line is erased. By analogy, a reference line in the OPUS notation is used to align other objects but does not appear in the final interface. The appearance of several reference lines along with their owning frame is illustrated in Figure 4. Reference lines come in several varieties and can be used to perform computations that aid in common alignment tasks. In addition to free reference lines whose position is determined by a single constraint, reference lines may also determine their position by computing minimums, maximums, and averages over a set of incoming constraints. These $\min$, max, and average reference lines can be used to build displays that expand to hold their contents and to perform centering operations. Finally, proportional reference lines are designed to maintain a proportional distance from their owning frames (e.g., a reference line that remains 10 percent of the way from the top of its frame independent of the frame size).

- Constraints are expressed using arrows as illustrated in Figure 5. Constraints between objects are expressed with a line starting with a circle at the anchor end and ending with an arrowhead at the constraint end (as illustrated by the top and center constraints of Figure 5). In addition, constraints can be anchored at a fixed point on the screen. These constraints begin with a diamond (as illustrated by the leftmost constraint of Figure 5). Each constraint arrow represents an equation relating one coordinate of the anchor object (or point) to a coordinate of the constrained object. By clicking on the constraint, the user can expose the exact equation used for the constraint (as shown by the center constraint of Figure 5). This equation can be edited to provide an expression for the constraint in a Pascal-like expression language, which prohibits side-effects. Within this expression, the symbol $\$$ is used to refer to the anchor coordinate value. As a consequence, since most constraints used in practice are offsets, many equations are of the form \$+value. Finally, the 

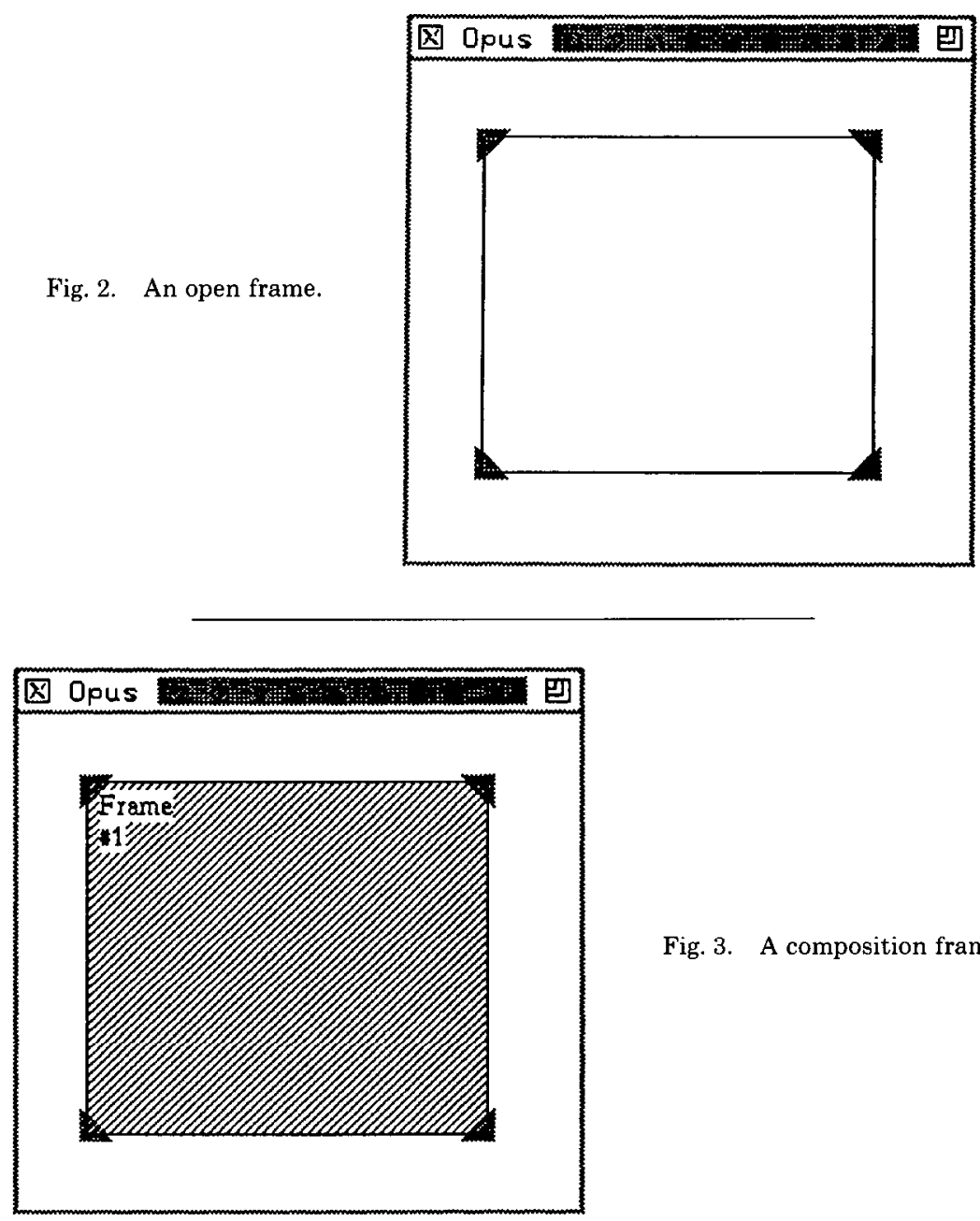

Fig. 3. A composition frame.

OPUS editor provides a special same-as operation that allows the user to designate that two or more constraints are to use the same equation always; whenever one of these equations is edited, they are all changed. This makes it easy to produce layouts that have uniform spacing between otherwise independent components.

-Interactor objects of various classes form the final component of the graphical notation. These are the only elements of the OPUS graphical notation that result in visible objects in the generated interface. Each interactor is represented by a characteristic icon. Currently the system supports the following interactor object classes (as portrayed from left to right in Figure 6): horizontal sliders, vertical sliders, rectangles, bitmaps (icons), text display and edit fields, push buttons, and two state (radio) buttons. In the future all ARTKit interactor classes will be supported. 


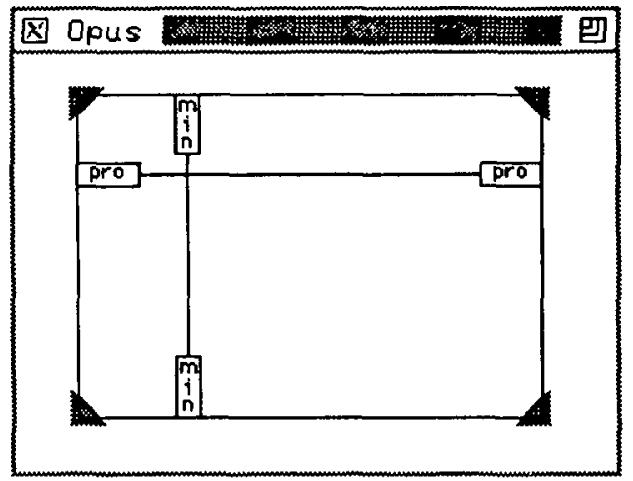

Fig. 4. A frame with several reference lines.

Fig. 5. Sample constraints.

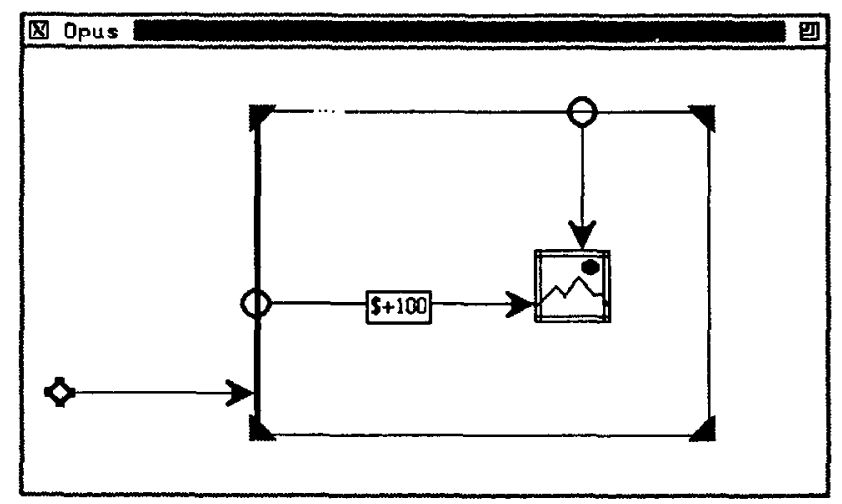

If any edge of an object is left unconstrained in the interface description, it is given an initial value of the screen position it occupies, but no equation is attached. Additionally, if it is associated with an interface object, the Penguims cell corresponding to the edge is made a controls parameter of that object. As a result, it is subject to input by the user (in most cases by dragging the object). This allows construction of some forms of new input devices within the system itself.

In addition to size and position, the OPUS editor allows the specification of other characteristics of interactor objects. These characteristics are specified by means of a property list. The property list interface is brought up by clicking on an interactor object. The interface consists of a form to be filled in by the user (as shown in Figure 7). If any fields are left blank, defaults are used in their place with the use of the Penguims keyword default. In addition to constant values, equations may also be entered into property list fields. This allows the full power of the Penguims system to be used in dynamically controlling the characteristics of each interactor. 


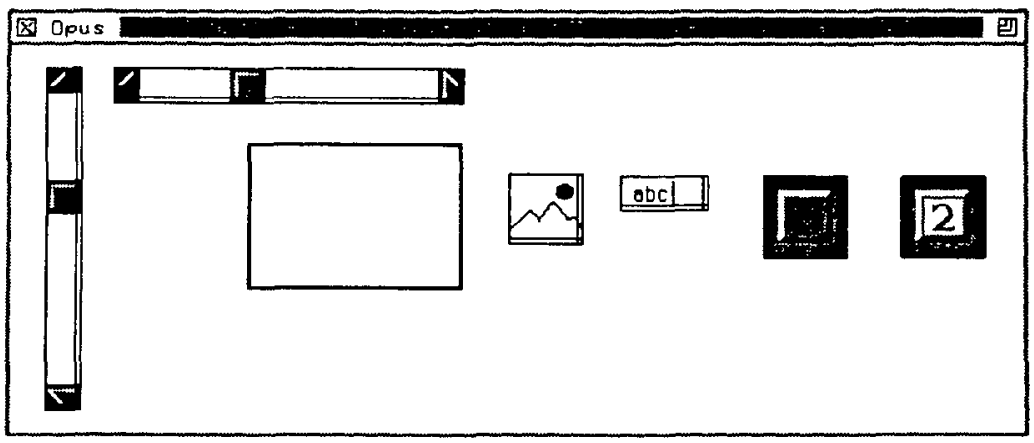

Fig. 6. Icons representing interactor objects.

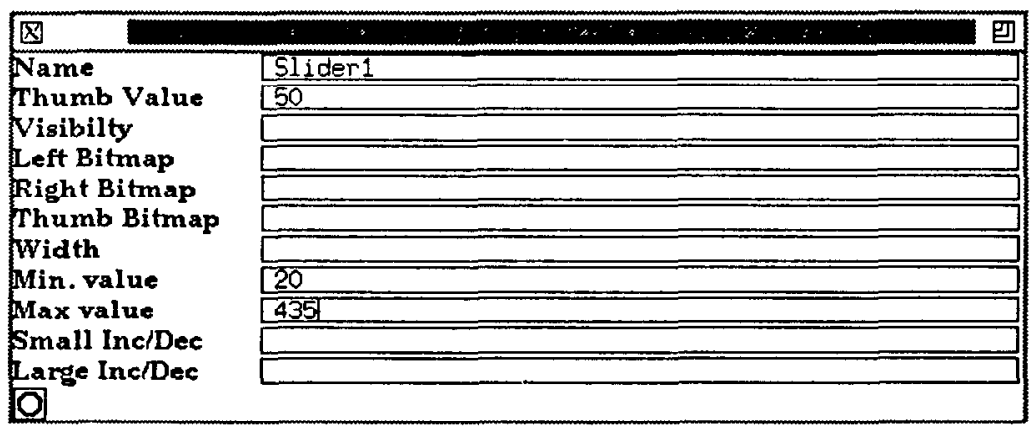

Fig. 7. A property list for a horizontal slider.

\subsection{Using the Notation}

The basic components of the graphical notation presented in the last subsection provide a powerful set of building blocks for describing a wide range of user interface layouts. It may not be readily apparent, however, how these building blocks can be combined to perform specific tasks. This subsection considers how the basic components of the notation can be used to express common layout constructs.

Figures 8,9 , and 10 illustrate three common layout descriptions. Figure 8 shows a simple layout with a rectangle that has a fixed size and is positioned a fixed distance from one corner of its reference frame. This form of layout is appropriate for a button or other fixed size interactor that need not expand or move when its surrounding environment changes.

Figure 9 shows a rectangle that stretches to fill the space available to it. This layout is appropriate for controlling a central display that should expand to make use of all available space. Figure 10 shows a rectangle that stretches to consume a fixed proportion of its owning frame. This form of layout is appropriate for a display that needs to share its space with other elements of the interface.

Finally, Figure 11 shows a more complex specification in which two rectangles are centered below another rectangle. This specification makes use of an average 


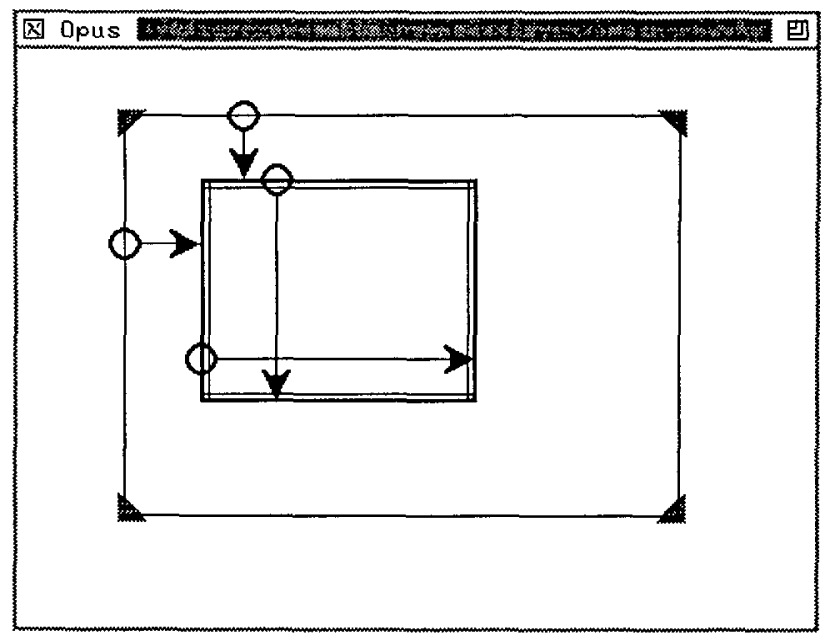

Fig. 8. A rectangle of fixed size that sticks to a corner.

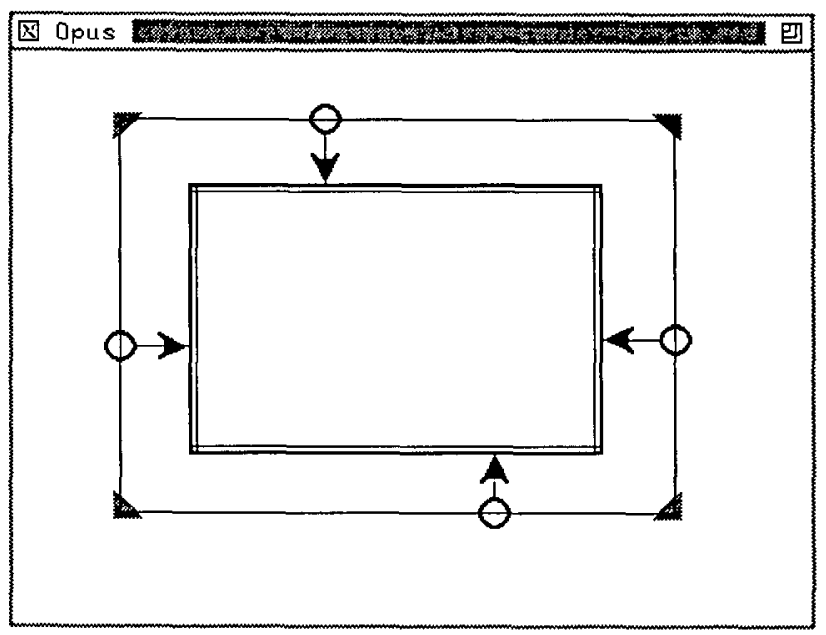

Fig. 9. A rectangle that expands to fill available space (fixed border).

reference line to establish a center point and uses the same-as operation to ensure that both sides of the rectangles are equidistant from this point.

Note that if the rectangles used in these examples were replaced by composition frames, the same descriptions could be used to compose smaller independent layout descriptions into larger presentations. To form these compositions, the enclosing frames of each of the smaller presentations is superimposed on the composition frames found in the overall presentation. This allows the direct graphical specification of composition operators that take smaller presentations as parameters [20]. 


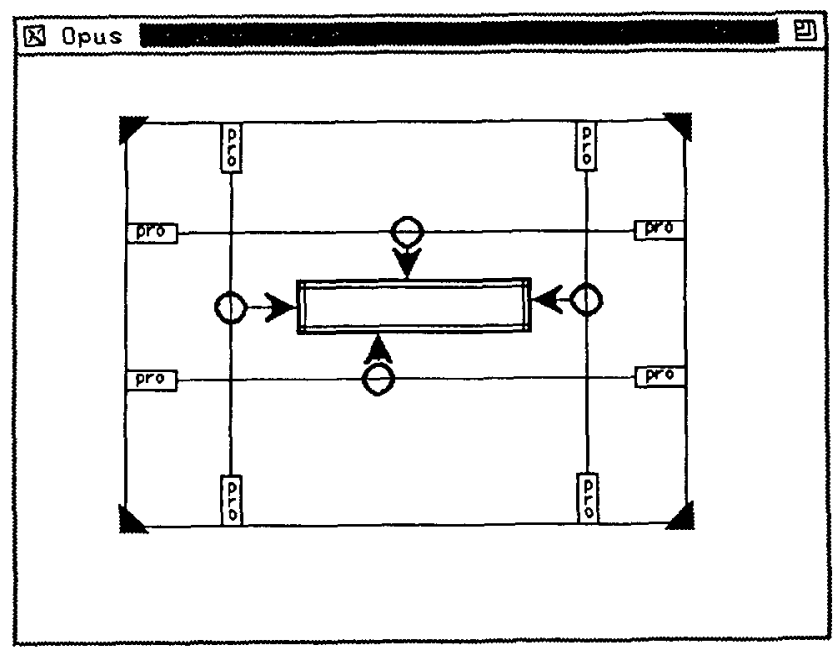

Fig. 10. A rectangle that stretches proportionally with the frame.

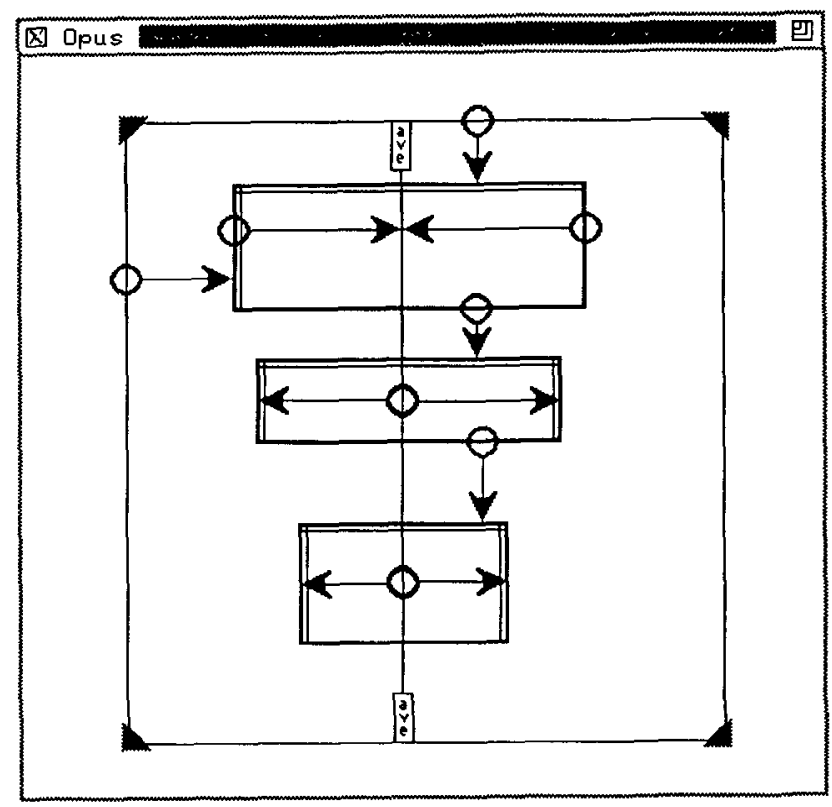

Fig. 11. A centering example.

An important aspect of Penguims generated user interfaces is their ability to support components that change size and position dynamically. Interfaces that must respond to changes in size often employ two strategies for layout, an outside-in strategy and an inside-out strategy. In the outside-in strategy, enclosing components (such as the overall window containing the display) are given a size, then the enclosed components must produce a display that conforms to these ACM Transactions on Information Systems, Vol. 8, No. 3, July 1990. 


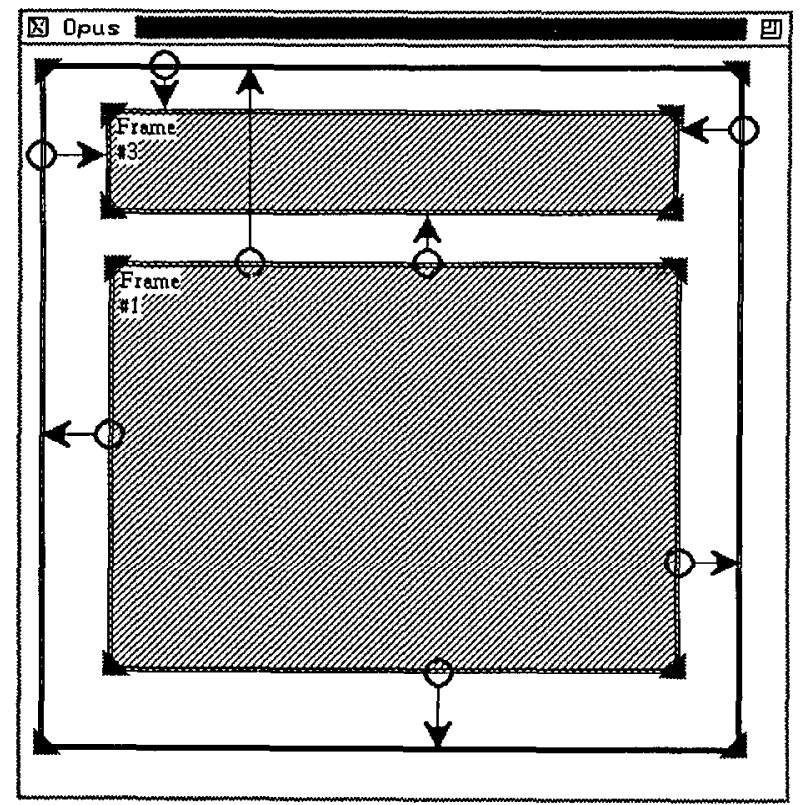

Fig. 12. A mixed inside-out, outside-in layout.

space requirements. This approach is used for each of the examples given above as well as all the interfaces generated by the system described in [6]. In the other strategy, inside-out, interior components define their natural size, and enclosing components expand or contract to conform to these requirements. Good layouts often require the use of both the inside-out and outside-in approaches. The Penguims/OPUS system allows both of these approaches to be integrated within one general mechanism for dynamic positioning and semantic feedback.

An example of mixed outside-in and inside-out layout is shown in Figure 12. Here, a large central frame determines the overall dimensions of the presentation in an inside-out manner, while a smaller frame at the top is controlled in an outside-in fashion. This would be appropriate for an important display (such as the canvas for a drawing program) placed below a row of buttons or other fixed interactors.

As a final example, Figure 13 shows an interface for a system performance tool. The major components of the OPUS specification for this interface is shown in Figure 14. In this case, the overall composed presentation is shown in the window at the top left. This presentation contains three subframes organizing the left column of buttons, the bar chart, and row of buttons at the bottom. Fach of these subframes in turn controls the placement of a row or column of labeled buttons or rectangles taken from the library as shown in the bottom right window.

This is an application that gathers and displays system performance statistics (e.g., CPU utilization, load average, page faults per second, etc.) for selected systems on our local area network. The machine whose statistics are to be displayed is selected by a set of radio buttons on the left. The bar chart presenting performance information is drawn by means of rectangles whose bases are 


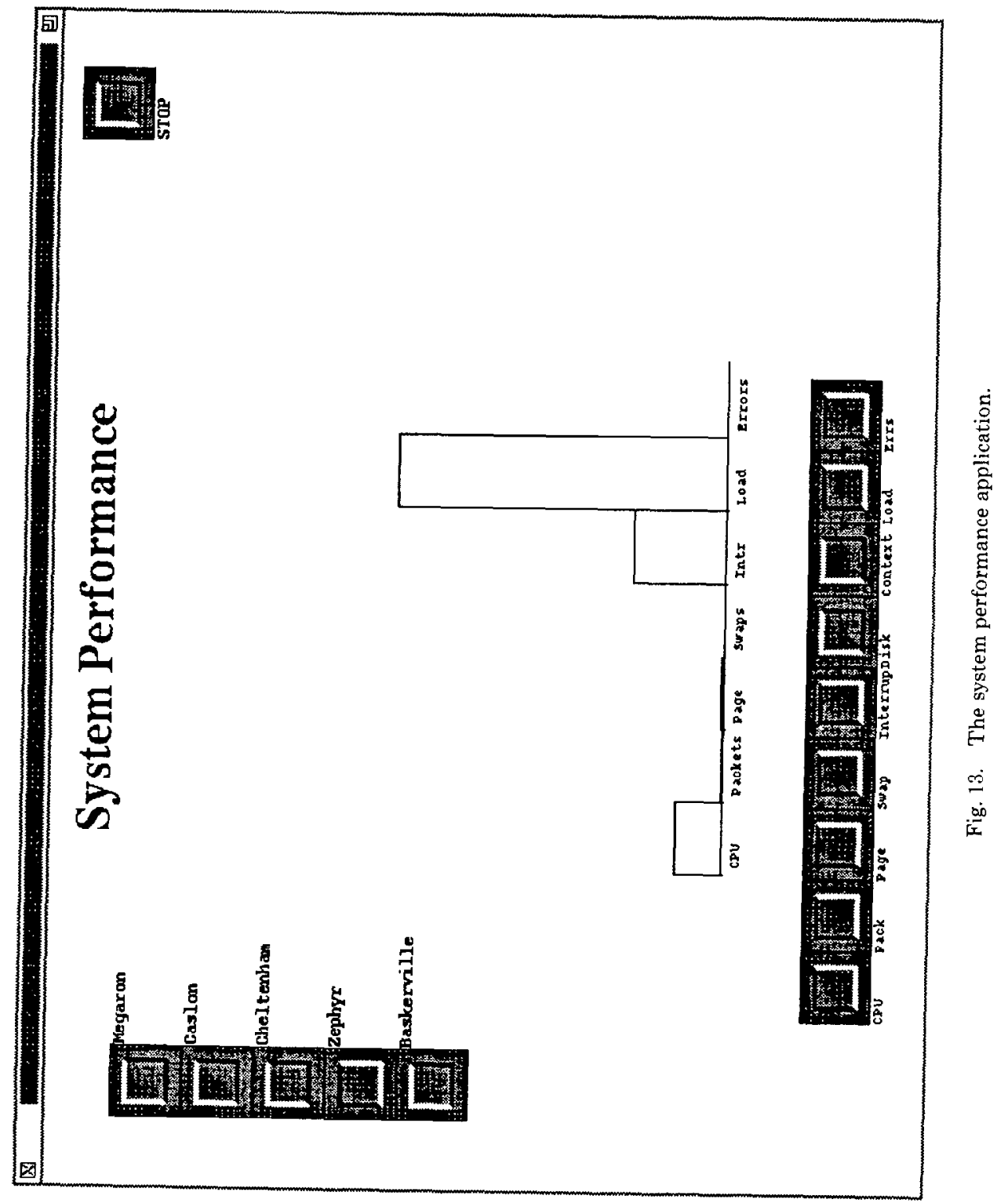




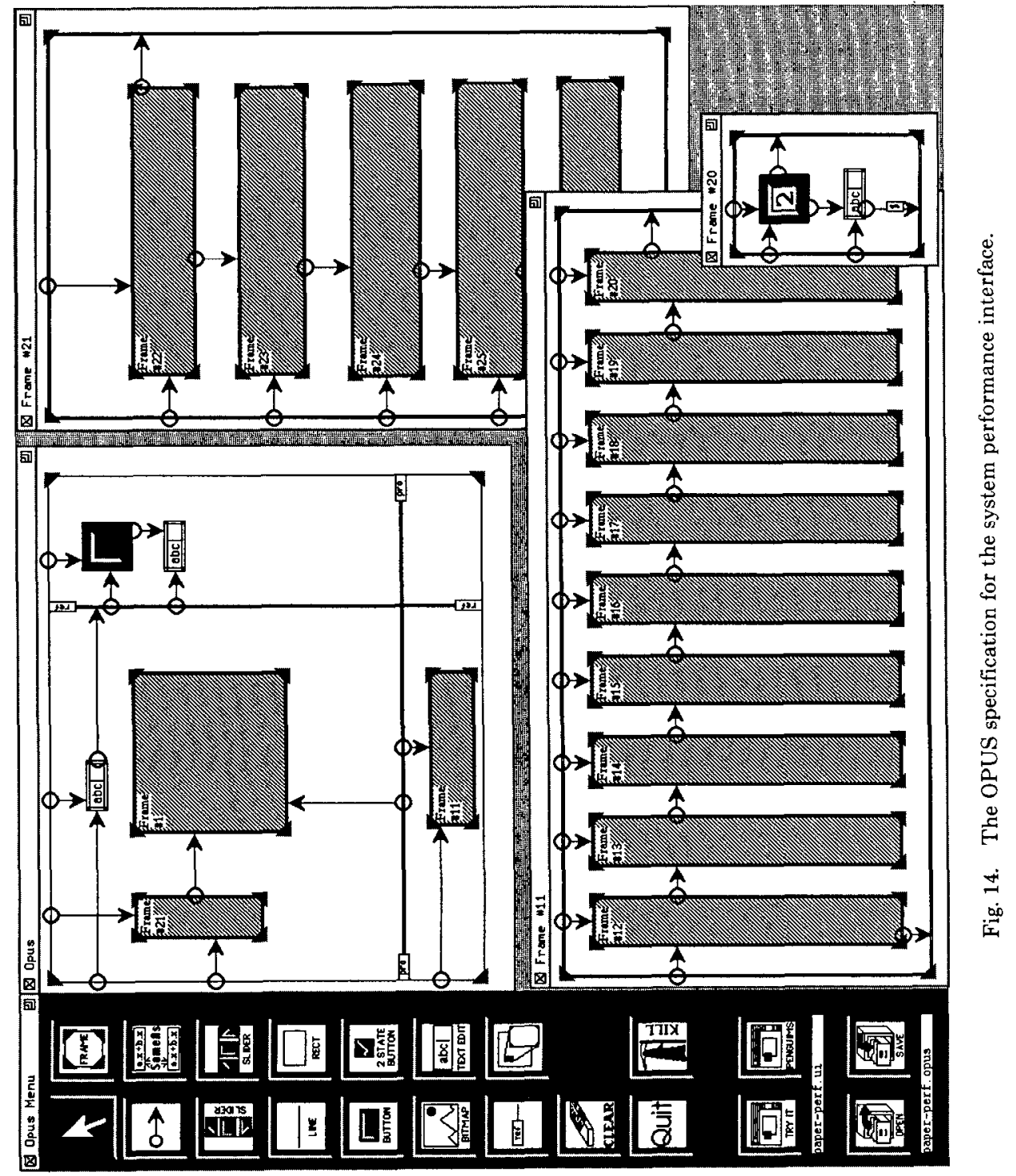


anchored to a reference line. The height of each rectangle is controlled by the value of the performance statistic it represents. Application routines to gather this information are linked with a version of the Penguims system and made available via Penguims function calls. These functions are then invoked directly from within constraint equations that control the height of each rectangle making up the bar chart.

To illustrate a situation where the user is given direct control over the configuration of the display, the visibility of each rectangle is controlled by one of the set of two-state buttons below the rectangles. This connection is accomplished by placing an equation in the visibility field of the property list of the rectangle. In addition, the left-hand edge of each rectangle depends on the righthand edge of the previous one. The width of each rectangle is derived using an equation of the form:

\section{if visible then $\$+40$ else 0}

Consequently, any undisplayed rectangles are removed from the display and the remaining rectangles automatically move over to close the gap. In the example we can see that the buttons for "Disk" and "Context" are up (off), while all the others are down (on); hence the rectangles for "disk" and "context" are not displayed while all others are.

\subsection{Views in the Editor}

As shown previously in Figure 1, the OPUS interface editor provides a palette of specification components much like a conventional drawing program. A view of each separate interface specification is presented in an individual window. Except for external constraints affecting its frame, each interface grouping may be manipulated independent of any composition it may be part of.

In addition to this program view of the specification, the editor also supports an execution view of the interface. This view is obtained by pressing the "try it" button. When the execution view is requested, the system generates specially modified Penguims code for the interface and internally invokes the Penguims UIMS on this code. Generation of this interface is essentially instantaneous (under $\frac{1}{2}$ second on a Sun SparcStation 1); consequently the user has the illusion of a seamless system despite the use of two separate programs. Unlike the final generated interface, internal positioning objects (frames and reference lines) are explicitly displayed in the execution view. This makes it easier to see the relationships among dynamically moving objects in the test interface in a debugging context.

The ability to instantly try out a partially specified interface with the execution view is very important since an explicit goal of the OPUS system is to support interfaces containing components that change size and position dynamically. In this situation it is often necessary to explore the dynamic behavior of the interface as well as its static specification.

Note that the program view of the interface does not attempt to evaluate constraints or place interface components in their actual screen positions. Experience with the system has shown that this design decision is the correct one. 
The program and execution views have different purposes and hence different layout requirements. In general, more space is needed to comfortably manipulate the interface specification than is needed in the interface itsclf. For example, many very short (often zero length) offset constraints are used in practice. These would be nearly impossible to manipulate if presented in actual size. In addition, frames and reference lines, which serve to organize and structure the specification, do not require space in the final interface.

\section{RELATED WORK}

Graphical specification of user interfaces in one form or another has been a topic of research starting with some of the earliest UIMS work [5] and continuing to this day (see for example $[9,16,22,31,32]$ ).

The OPUS system concentrates primarily on the presentation aspects of the interface. A primary contribution of the system is its ability to deal with the dynamically changing size and position of its components in a flexible manner. The initial work on the OPUS notation was inspired by the dialog editor tool described in [6]. This system was one of the first to deal with the specification of layouts that could respond intelligently to dynamic size changes. It employs a very simple graphical notation but has much less descriptive power than the OPUS notation (equivalent to the use of only proportional reference lines and constant offset constraints). In addition, the system only supports outside-in layouts and does not provide a specification composition mechanism.

Other systems have a close relationship with OPUS and the Penguims UIMS by virtue of their underlying execution model as well as their graphical specifications. Early work involving the use of more general multiway constraints in a graphical framework includes the ThingLab and Animus Systems [1-3, 7]. These systems support a general graphical notation for constraints, but do not provide a specialized notation for common cases or a convenient means for composition. A related notation is employed in the InterCONS system [33] for describing interfaces built with data-flow programs.

Perhaps the most closely related work is that of the Lapidary system [23], which is part of the Garnet user interface development environment [24]. This system provides an underlying one-way constraint satisfaction system similar to that provided by the Penguims UIMS and provides a graphical interface for describing interface layouts based on these constraints. The primary difference with the OPUS system is in the notational approach taken by the Lapidary system - it attempts to create interfaces primarily by demonstration. The interface designer is only shown the final appearance of the interface, and no notation is provided for constraints once they have been specified.

\section{EXPERIENCE, LIMITATIONS, AND FUTURE WORK}

While the OPUS system has not been used for a large interface project, we have gained significant experience using it to construct smaller interfaces. This experience has helped refine the system through three versions; more formal user testing to measure productivity and identify remaining weaknesses is planned. 
One practical outcome of this experience has been the addition of a hierarchical structuring mechanism (composition frames). Without hierarchical structuring, the OPUS notation suffers from a problem common to many visual programming systems-large specifications become confusing and are difficult to understand. The addition of composition frames in the last version has made it possible to deal with larger designs in a more understandable and structured fashion. Of particular importance has been the ability to create a library of reusable pieces that can be incorporated into larger interfaces.

Our experience with the system has also shown that one-way constraint systems are a powerful mechanism for expressing layout relationships and that nearly all the constraints needed in practice can be expressed within the graphical notation (without resorting to complex textual expressions). The one exception to this has been in expressing some more complicated centering relationships. We are currently exploring new notational constructs specifically designed to express notions of centering.

Finally, a current limitation of the OPUS editor is the lack of support for number independent layouts. At present, displays with a dynamically changing set of component objects must be implemented in Penguims (although most subpieces can be specified in OPUS). We are currently exploring graphical specification techniques related to the graphical search and replace system described in [17] to overcome this problem. This technique would allow new components to be added to existing structures in a manner similar to generative graph grammars, but with the support of a graphical notation to describe production rules.

\section{CONCLUSIONS}

This paper has described the concepts and implementation of the OPUS user interface editor. This system works with the Penguims UIMS, which is based on incremental evaluation of one-way constraints-a computational model closely related to the one used in spreadsheets. The OPUS system allows a wide variety of size and position independent layouts to be specified in an easy-to-use graphical notation with little or no explicit programming.

\section{ACKNOWLEDGMENT}

The authors would like to thank Tyson Henry who implemented parts of the first version of the OPUS system.

\section{REFERENCES}

1. Borning, A. The programming language aspects of ThingLab, a constraint-oriented simulation laboratory. ACM Trans. Program. Lang. Syst. 3 (Oct. 1981), 353-387.

2. Borning, A. Defining constraints graphically. In Proceedings of $C H I$ ' 86 (Boston, Mass., April 1986), 137-143.

3. Borning, A., AND Duisberg, R. Constraint-based tools for building user interfaces. $A C M$ Trans. Gr. 5 (Oct. 1986), 345-374.

4. Borning, A., Duisberg, R., Freeman-Benson, B., Kramer, A., and Woolf, M. Constraint hierarchies. In Proceedings of OOPSLA '87 (Orlando, Fla., Oct. 1987), 48-60. 
5. Buxton, W., Lamb, M., Sherman, D., and Smith, K. Towards a comprehensive user interface management system. In Proceedings of SIGGRAPH '83 (Detroit, Mich., July 1983), 35-42.

6. Cardel.t. T. Building user interfaces by direct manipulation. In Proceedings of the ACM SIGGRAPH Symposium on User Interface Software (Banff, Alberta, Oct. 1988), 152-166.

7. Duisberg, R. Animating graphical interfaces using temporal constraints. In Proceedings of $\mathrm{CHI}$ '86 (Boston, Mass., April 1986), 131-136.

8. Goldberg, A., And Robson, D. Smalltalk-80: The Language and its Implementation. AddisonWesley, Reading, Mass., 1983.

9. Henderson, D. A. The trillium user interface design environment. In Proceedings of CHI' 86 (Boston, Mass., April 1986), 221-227.

10. Henry, T. R., Hudson, S. E., ANd Newell, G. L. Integrating gesture and snapping into a user interface toolkit. To appear in Proceedings of the ACM SIGGRAPH Symposium on User Interface Software and Technology (Snowbird, Utah, Oct. 1990).

11. Hudson, S. E. UIMS support for direct manipulation interfaces. ACM Trans. Comput. Gr. 21, 2 (Apr. 1987), 120-124.

12. Hudson, S. E. Semantic feedback in the Higgens UIMS. IEEE Trans. Softw. Eng. 14, 8 (Aug. 1988), 1188-1206.

13. Hudson, S. E. Incremental attribute evaluation: A flexible algorithm for lazy update. Tech. Rep. TR 89-12, Dept. of Computer Science, Univ. of Arizona (submitted for publication).

14. Hudson, S. E. Adaptive semantic snapping-A technique for semantic feedback at the lexical level. To appear in Proceedings of CHI'90 (Seattle, Wash., April 1990).

15. Hutchins, E. L., Hollan, J. D., AND Norman, D. A. Direct manipulation interfaces. In User Centered Systems Design, D. A. Norman and S. W. Draper, Eds. Lawrence Erlbaum Associates, Hillsdale, N.J., 1986, pp. 87-124.

16. JACOB, R. J. K. A specification language for direct manipulation user interfaces. ACM Trans. Gr. 5, 4 (Oct. 1986), 283-317.

17. Kurlander, D., ANd Bier, E. A. Graphical search and replace. ACM Trans. Comput. Gr. 22, 4 (Aug. 1988), 113-120.

18. Let.er, W. Constraint Programming Languages: Their Sperification and Generation. AddisonWesley, Reading, Mass., 1988.

19. LEwIS, C. H. NoPumpG: Creating interactive graphics with spreadsheet machinery. Tech. Rep. CS-CU-372-87, Univ. of Colorado, Aug. 1987.

20. Linton, M. A., Vlissides, J. M., And Calder, P. R. Composing user interfaces with InterViews. IEEE Comput. 22, 2 (Feb. 1989), 8-22.

21. MCCormack, J., ANd Asente, P. An overview of the X Toolkit. In Proceedings of the ACM SIGGRAPH Symposium on User Interface Software (Banff, Alberta, Oct. 1988), 46-55.

22. Myers, B. A., AND Buxton, W. Creating highly interactive graphical user interfaces by demonstration. ACM Trans. Comput. Gr. 20, 3 (Aug. 1986), 249-258.

23. Myers, B. A., VAnder Z $\Lambda$ nden, B., AND DANnenberG, R. B. Creating graphical interactive application objects by demonstration. In Proceedings of the ACM SIGGRAPH Symposium on User Interface Software and Technology (Williamsburg, Va., Nov. 1989), 95-104.

24. Myers, B., Giuse, D., Dannenberg, R., Vander Zanden, B., Kosbie, P., Pervin, E., and Kolojejchick, J. The Garnet toolkit reference manuals: Support for highly-interactive, graphical user interfaces in Lisp. CMU Tech. Rep. CMU-CS-89-196, Computer Science Department, Carnegie Mellon University, Nov. 1989.

25. Scheifler, R. W., AND Getrys, J. The X window system. ACM Trans. Gr. 5 (Apr. 1986), $79-109$.

26. Schulert, A. J., Rogers, G. T., And Hamilton, J. ADM-A dialog manager. In Proceedings of CHI'85 (San Francisco, Apr. 1985), 177-183.

27. Shneiderman, B. The future of interactive systems and the emergence of direct manipulation. Behav. Inf. Technol. I (1982), 237-256.

28. Shneiderman, B. Direct manipulation: A step beyond programming languages. Comput. 16 (Aug. 1983), 57-69.

29. Szekely, P. A., AND Myers, B. A. A user interface toolkit based on graphical objects and constraints. In Proceedings of OOPSLA '88 (San Diego, Calif., Sept. 1988), 36-45.

30. Sun Microsystems Inc. Sun View 1 Programmer's Guide. Sun Microsystems Inc., Mountain View, Calif, 1988. 
31. Singh, G., And Green, M. Designing the interface designer's interface. In Proceedings of the ACM SIGGRAPH Symposium on User Interface Software (Banff, Alberta, Oct. 1988), 109-116.

32. SiNGH, G., AND GrEeN, M. Chisel: A system for creating highly interactive screen layouts. In Proceedings of the ACM SIGGRAPH Symposium on User Interface Software and Technology (Williamsburg, Va., Nov. 1989), 86-94.

33. Smith, D. N. Building interfaces interactively. In Proceedings of the ACM SIGGRAPH Symposium on User Interface Software (Banff, Alberta, Oct. 1988), 144-151.

34. Wilde, N., AND Lewis, C. H. Spreadsheet-based interactive graphics: From prototype to tool. In Proceedings of CHI'90 (Seattle, Wash., Apr. 1990), 153-159. 